\title{
EL NO-LUGAR DE LA TEORÍA LITERARIA
}

\author{
Eduardo Subirats* \\ New York University
}

Resumen: En el siguiente documento se presenta un crítica a la cultura literaria contemporánea partiendo del análisis de la obra $O$ lugar da teoría literaria (Andre Cechinel), en la que se plantea el mercado de la cultura como caso de "servidumbre intelectual voluntaria", instaurada a partir de la auto-negación de la conciencia reflexiva. Todo lo anterior a partir tres obras ejemplares de la conciencia intelectual del siglo veinte: Molloy, Der Process y Pedro Páramo. Se centrará en la categoría subjetiva y ontológica de voluntad, teniendo en cuenta la voluntad artística como la esencia de la obra de arte. Así las cosas, la teoría, asunto central dentro de esta crítica, sería un devenir consciente de la voluntad artística de comprender y transformar el mundo.

Palabras clave: Servidumbre intelectual; voluntad; posliteratura; Weltliteratur; crisis contemporánea

Recibido: 14 de septiembre de 2016

Aprobado: 21 de noviembre de 2016

\section{THE NO-PLACE OF THE LITERARY THEORY}

Abstrac: The next document presents a critic of the contemporary literary culture starting by the analysis of the work O lugar da teoría literaria (Andre Cechinel), in which it raises the market of the culture as the case of "voluntary intellectual serfdom", established after the selfnegation of the reflexive consciousness. All this since three exemplary works of the intellectual consciousness of the XX century: Molloy, Der Process and Pedro Páramo. It focuses in the subjective and ontological category of will, taking in account the artistic will as the essence of the piece of art. As a matter of fact, the theory, main subject of this critic, would be an aware becoming of the artistic will of the understanding and transforming the world.

Key words: intellectual serfdom; will; post-literature; Weltliteratur; Contemporary crisis.

*Eduardo Subirats. Filósofo y ensayista nacido en Barcelona. Ha escrito numerosos libros, entre los que se destacan El continente vacio: la conquista del nuevo mundo y la conciencia moderna (1994) y el monumental Mito y literatura (2014). Es profesor en New York University y en la Escola da Cidade, Facultad de Arquitetura de São Paulo. 
Me acaba de llegar por correo postal un libro de mis amigos de Brasil. Su título es ligeramente ampuloso para nuestra edad convulsa: $O$ lugar da teoría literaria. No solamente vindica para la teoría un espacio administrativo en la organización corporativa del conocimiento. Al mismo tiempo, la levanta como fetiche de un único e irrebatible acceso a la verdad o, al menos, a una verdad literaria.

El libro en cuestión es también un síntoma del achicamiento del estructuralismo en un ritual administrativo de identidad en las últimas provincias del mundo global, mientras en las metrópolis ha degenerado en sistema académico de control y vaciamiento intelectual de las Humanities. Por lo demás, su introducción es todo un "Prólogo en el cielo": la crónica de una entrada triunfal de Jameson en Beijing. Tras esa apoteosis le sigue un compendio de papers tediosos que repiten como letanías los slogans de Lacan y Levy Strauss, y de Foucault, Derrida y tutti quanti...

No juzgo el provincianismo que supone glorificar la conquista de Tiananmem bajo la bandera del postmodern norteamericano en 2016 desde una perspectiva brasileira como si se tratara del advenimiento de una redención celestial. No lo considero provinciano simplemente porque en el global village no se puede distinguir en términos de intensidad intelectual a una tediosa provincia de su monótona metrópoli. En el mundo global todo se ha trivializado a los niveles pueblerinos de un village globalmente diseminado. Más bien juzgo esa adoración beata a cualquiera que se ponga en venta como redentor en el manipulado mercado de la cultura como un caso de servidumbre intelectual voluntaria.

Esta servidumbre es un fenómeno que se expande sin fronteras tanto entre las ex-naciones poscolonizadas, cuanto en las ex-metrópolis, bajo la tutoría de las corporaciones de propaganda académica y científica. Es una servidumbre instaurada a partir de la autonegación de la conciencia reflexiva y soberana, algo que la academia postmoderna ha legitimado sumariamente bajo las divisas del "final del sujeto", "final de la autoconciencia moderna" y final de los grands récits. Es también una servidumbre en términos de inmolación monacal de la conciencia individual, en aras a los principios de la obediencia, la repetición y la 
abdicación de la propia experiencia. Esta servidumbre voluntaria y postcolonial presupone la renuncia a reconocerse a sí mismo en una literatura, una mitología y unos dioses propios; y de reconocerse en una tradición intelectual. La deserción del principio esclarecedor que formuló Horacio: “¡Sapere aude!”.

Lo primero que debería llamar la atención en esas compilaciones locales, y en los tratados globales de Literary Theory o Postheory en los que se inspiran, es nada más y nada menos que la ausencia de toda referencia a una obra de arte, ya sea musical, literaria o visual; y la ausencia de una referencia tanto a literaturas regionales cuanto a un concepto reflexivo de Weltliteratur, una literatura mundial.

Esta ausencia de referentes es una condición institucional para la iniciación ritual a los cursos de Introduction in the Literary Theory. Es la premisa epistemológica de un estructuralismo cuya real función no es la capacitación reflexiva del estudiante a través de la lectura literaria en una sociedad progresiva e intencionalmente iletrada. Su objetivo sistémico consiste en la reducción de toda obra artística a lenguaje, y su degradación ontológica y psicológica a ficción. Y la fetichización de la Teoría es la contraparte de esta reducción lingüística de la literatura a una subcategoría de consumo cultural. Su consecuencia final: la celebración del "último libro", y la postliteratura y el postarte en una edad posthumana.

Pero también me llama la atención la doble ceguera de esta teoría fetichista frente a la ostensible circunstancia de que no existe un análisis consistente de obras literarias clásicas y modernas del siglo veinte en América latina, ni de sus analogías y diferencias con otras obras máximas de la literatura mundial, ni menos todavía sus relaciones cruzadas con otras actividades artísticas e intelectuales. Me llama la atención la insensibilidad de los mentores de estas teorías prêt-à-porter frente a la intensidad de los vínculos entre la literatura, la música y la pintura, la arquitectura y el cine latinoamericanos, su permanente diálogo con lenguas y mitos milenarios, y su integración de experiencias espirituales y mágicas. No puedo dar crédito a su ceguera frente a la visión trágica de la historia colonial latinoamericana como un proceso de permanente empobrecimiento teológico-político en obras tan fundamentales como las de Roa Bastos, Juan Rulfo o José María Arguedas. 
Pero no he venido aquí a levantar testimonio del estado decrépito de los estudios literarios y humanísticos en Europa, Latinoamérica y los Estados Unidos. Deseo hablarles precisamente de todo lo contrario. Quiero hablarles de la reflexión teórica sobre literatura, de diálogo entre filosofía y poesía, de la interacción mitos y novelas. Les quiero hablar de las inagotables dimensiones cognitivas y espirituales que encierra toda verdadera obra de arte. Desearía poder hablarles asimismo del conflicto entre esta tradición humanista y reflexiva en la que pueden citarse a partes iguales Rabindranath Tagore, Thomas Mann y João Guimarães Rosa, y por el lado opuesto y contrario la institucionalización logocéntrica y perfectamente cínica de la escolástica estructuralista en las redes académicas globales.

El primer paso necesario para abrirnos a esta multiplicidad de aspectos interrelacionados es definir esta reflexión literaria y replantear una visión filosófica sobre la literatura y las artes. Y lo haré en los términos generales de un espacio de encuentro entre las categorías filosóficas y las narraciones mitológicas y literarias. Un espacio ideal entre poesía y filosofía que podemos crear, por poner un ejemplo, si juntamos la conciencia moderna de una regresión cultural y científica formulada a la vez por Simmel y Einstein, y la conciencia mitológica de la unidad espiritual del humano con una naturaleza mágica y fructífera en la poética de Arguedas y Rulfo, o en la pintura de Tarsila do Amaral o Toledo. Es un espacio reflexivo sin fronteras entre lo creativo y lo reflexivo, y entre la expresión poética y la reconstrucción crítica.

Por otra parte, no podemos estar hablando de teoría literaria sin antes saber qué clase de literatura estamos leyendo: el Ramayana o la ficción comercial de Vargas Llosa. Sin hacer estas distinciones formales y de contenido caeríamos en la misma pretensión estructuralista de no querer ver por el telescopio de Galileo el movimiento no-geocéntrico del cosmos porque no era consonante con las jergas políticamente correctas de la escolástica pontificia. Por esto mencionaré a tres obras ejemplares de la conciencia intelectual del siglo veinte: Molloy, Der Process y Pedro Páramo. Tres obras representativas de la literatura mundial, de la Weltliteratur en el sentido que Goethe dio a esta palabra (no en el sentido uniformado por una lingüística trascendental bajo la jurisdicción de una literatura "comparada"). 
Molloy de Beckett resume una crítica radical de la decadencia de la civilización europea y pone punto final a la novela occidental a partir de la disolución de los presupuestos epistemológicos, éticos y políticos del narrador y la narración. Pedro Páramo de Juan Rulfo: el relato terminal del proceso de destrucción colonial latinoamericano representado por sus dos grandes instrumentos políticos: el cacique y el sacerdote. Pedro Páramo también pone de manifiesto el final del narrador. Pero un final del sujeto de la narración que ahora no tiene lugar a lo largo de una desarticulación esquizofrénica y autista del sujeto trascendental, como en las novelas de Beckett, sino a través del retorno a un pasado mitológico y a una unidad no dualista de la vida y la muerte en la civilización azteca. Der Process de Franz Kafka representa la claudicación del humano frente a un poder burocrático totalitario que, sin embargo, está constituido como sistema racional de una Ley sagrada, absoluta y verdadera.

Quiero presentarles a continuación una categoría estética fundamental para comprender estas obras: la categoría subjetiva y ontológica de voluntad. Quiero poner el acento sobre la voluntad artística como la esencia de la obra de arte; y quiero subrayar la voluntad estética que reúne una conciencia del pasado y el presente, y una visión de futuro en una obra literaria o una obra de arte: las tres dimensiones temporales del conocimiento de la memoria representada por Mnemosyne.

No tengo que recordarles que este concepto de voluntad tiene una historia. Schopenhauer lo utilizó decididamente contra el formalismo de la estética kantiana. Su definición de la voluntad como la unidad de sujeto y objeto, o de la conciencia y el ser, le llevó a identificarlo con el eidos griego. La voluntad es la Idea, la Realidad absoluta. Nietzsche extendió más tarde este concepto de voluntad a sus raíces mitológicas y místicas que vinculan la tragedia griega con el ritual ditirámbico. Lo único que debemos añadir hoy a este concepto mitológico, metafísico y psicológico de voluntad es la conciencia expresionista de fragmentación, destrucción y vacío que impregna a esta voluntad artística en nuestro siglo: en Kafka, Rulfo y Beckett, entre otros.

Tampoco tengo que subrayar su carácter polémico con respecto a las estrategias institucionales de la muerte del sujeto, la desaparición del 
intelectual y el último artista. Al poner de manifiesto en primer plano de la crítica literaria el concepto de voluntad llamo claramente la atención sobre su centralidad, como categoría a la vez subjetiva y objetiva, en toda obra de arte, incluso o precisamente allí dónde esta obra literaria o artística ponga reflexivamente de manifiesto su derrumbamiento. Es el caso de los tres escritores que he señalado.

Seguidamente podemos subdividir esta voluntad estética en cuatro apartados. Y seguiré el ejemplo de Pedro Páramo porque todos ustedes conocen esta novela. Primer nivel lingüístico: la estructura de esta novela se articula en torno a dos narradores, Juan Preciado y Susana San Juan; y la narración reconstruye un proceso progresivo de desarticulación a la vez lingüística y humana.

Segundo, el punto de vista religioso y mitológico: tiempo y espacio de la novela son al mismo tiempo simbólicos y descriptivos; son tiempos y espacios mitológicos y, simultáneamente, espacios racionales y realistas. El relato oscila entre el cacique y el sacerdote como unidad político-teológica colonial, y las mujeres, psicológica y profundamente identificadas con las diosas aztecas y zapotecas de la tierra, y con los ciclos de la vida y la muerte que representan, o con las diosas celestiales tanto aztecas, como cristianas. La teología política de la culpa desempeña un papel fundamental en la constitución de una barrera mitológica y sexual entre el poder masculino y colonial, y la existencia de las mujeres como diosas degradadas del Mitlán, el reino subterráneo de la reproducción vegetal.

En tercer lugar es preciso considerar la voluntad psicológica, la voluntad como momento constituyente de la conciencia y la existencia individual humana. En esta novela de Rulfo, como también en las de Kafka o Beckett, esta voluntad aparece bajo el aspecto negativo de su desintegración. Una voluntad desintegrada que se pone de manifiesto en la historia de sus personajes damáticos principales, de Juan Preciado como paradigma de escisión psicótica de la personalidad, y el Padre Rentería como modelo de conciencia esquizofrénica. Como tal voluntad rota se opone a las normas constituyentes de sujetos racionalizados representantes del mundo moderno.

El cuarto apartado comprende el significado espiritual de toda auténtica obra de arte. Comprende su voluntad en el sentido metafísico 
de Idea. Una voluntad y un ideal enteramente negativo en una obra de Juan Rulfo, que revela esclarecedoramente los conflictos políticos, religiosos y militares del México postcolonial y del mundo.

Otro tanto puede decirse de la novela de Kafka. Gramaticalmente y existencialmente gira en torno a la degradación, la humillación y la destrucción de la conciencia moderna. Desde un punto de vista religioso su problema central es la Ley. Es la confrontación del humano y el orden ético la ley que define histórica y espiritualmente a una comunidad espiritual. Pero, en tercer lugar, esta visión metafísica comprende también una mirada política como las dos caras de una misma moneda: esa misma Ley sagrada y verdadera preside un sistema burocrático irracional, totalitario y corrupto.

Y lo mismo vale para Molloy de Beckett. Aquí el papel de la mitología lo desempeña la metafísica de Berkeley y Descartes. Ambos presiden un mundo completamente deshumanizado bajo los que la conciencia moderna se desarticula interiormente y desaparece.

Esos tres ejemplos literarios son paradigmáticos desde un punto de vista de su conciencia psicológica y su voluntad literaria: Kafka o el autismo; Beckett y la esquizofrenia; Rulfo y el regreso paranoico de la conciencia colonial derrotada al reino de las diosas ctónicas de la vida y la muerte. Tres delirios psicóticos de nuestro tiempo. Son también tres expresiones de la disolución de la voluntad como orden ético y ontológico del mundo que llamamos moderno: tres expresiones de una voluntad que se destruye a sí misma.

No necesito perder tiempo con largos argumentos para poner en evidencia que no puede establecerse ninguno de estos tres juicios estéticos sobre la esencia de estas obras a partir del grand récit de lingüísticas postsubjetivas y anhistóricas del estructuralismo. Los grandes hitos de langue y parole, las recetas del discourse o la narrativity, o las ilusiones metafísicas de writing y performativity simplemente ignoran esta dimensión profunda que he resumido a partir de una conciencia crítica de la crisis contemporánea y una teoría estética que he resumido bajo el concepto de voluntad.

He tratado de definirles someramente la teoría literaria como una hermenéutica. Leemos e interpretamos las obras literarias, la historia de 
los mitos y las ideas como si decodificásemos una ruina arqueológica. Ahora puedo añadir que la teoría literaria comprende una lingüística, una mitología y un principio filosófico y racional esclarecedor que permite la apertura de la conciencia a sus memorias originarias y a una reflexión sobre el presente, el pasado y el futuro. La teoría comprende, por este motivo, la mediación reflexiva entre la voluntad esencial de una obra de arte y su voluntad transformadora. La teoría es el devenir consciente de una voluntad artística de comprender y transformar el mundo.

La palabra griega theoria arraiga etimológicamente en la visión, en la percepción sensible y la apercepción intelectual de las cosas. En su República Platón define esta theoria como una contemplación que entrelaza la reflexión filosófica con la computación empírica. Una teoría es un sistema intelectual capaz de comprender las cosas del cielo y de la tierra en una visión unitaria, coherente y rigurosa. En este sentido nos referimos a los grandes sistemas filosóficos de Kant o Hegel como teorías.

Es ostensible que hoy esa theoria ha disminuido su alcance a la parvedad y parquedad de una microepistemología de aplicación local. Tenemos una teoría del global warming o la teoría queer. La posteoría literaria también ha sufrido está clase de recortes internos y ha ido menguando su horizonte intelectual hasta su completo eclipse.

No voy a entrar en la inútil discusión sobre las retóricas comerciales y las pasiones humanas que identifican estas microteorías como avantgar de revolucionaria. Sabemos o deberíamos saber el origen político de esta degradación de las Humanidades. Lo que articulaba las protestas revolucionarias en torno al año de 1968 eran teorías globales, sistemas conceptuales y políticos coherentes y consistentes, que vinculaban entre sí múltiples variables conceptuales. Esta articulación lógica se ha disuelto interna y externamente en un proceso electrónico de deconstrucción social.

He mencionado tres obras y tres delirios psicóticos desde esta perspectiva hermenéutica sobre la voluntad artística: Kafka o la paranoia, Beckett y la esquizofrenia, Rulfo y una psicosis regresiva de identificación con desmoronados mitos fundacionales. Quiero acabar esta reflexión sobre filosofía y literatura con un mito y un personaje 
literario afirmativo, que no acepta fronteras ni límites, que da expresión a una verdadera voluntad lingüística y ontológicamente afirmativa y creadora, que es un héroe civilizador y revolucionario, y que es el representante por antonomasia de una sexualidad fálica: Hermes, el trickster. Y tres novelas que expusieron expresamente la vida de tres aventureros y tres representantes modernos de la tradición picaresca del trickster: El idiota de Dostoievski, Macunaíma de Mário de Andrade y Feliz Krull de Thomas Mann.

El idiota es la visión reveladora de una decadente aristocracia rusa a través de la biografía de un aventurero psicótico y amante místico de las mujeres. Macunaíma es un dios al que todavía se venera en las culturas originales de Amazonia. Mário de Andrade lo transformó en un héroe literario anárquico, espiritual y asimismo sexualmente vinculado a todas las mujeres. Un héroe transgresor y, al mismo tiempo, mágico y cómico como un payaso sagrado. Thomas Mann eligió a Felix Krull como un joven intelectual y sexualmente identificado con el trickster Hermes. Y como pícaro, ladrón y aventurero Krull abandona una Europa destruida por las sucesivas guerras que ha atravesado en el siglo pasado y, a lo largo de una serie de aventuras sociales y amorosas, que Mann no pudo terminar, exalta la libertad, y el placer de vivir en sus colores más brillantes.

Menciono estas tres novelas porque definen un proyecto iluminador y un sueño de libertad. Todas ellas expresan un deseo erótico de aventuras y conocimiento. Todas parten de una visión religiosa y mitológica arcaica. Las tres novelas confrontan esta trascendencia espiritual del trickster con un mar de ruinas: una sociedad corrupta, ciudades bombardeadas y pueblos masacrados en silencio.

La reflexión teórica, el dialogo entre las filosofías, las religiones y las artes, levanta hoy, frente al abismo de la crisis histórica que estamos viviendo, esta voluntad originaria y fundadora, $\mathrm{y}$ transgresora y creadora: como El idiota, Macunaíma y Felix Krull. Repito: un concepto de voluntad que arraiga en el eidos platónico, y que Schopenhauer, Wagner y Nietzsche definieron musical, mitológica y ontológicamente. Y una voluntad que precisamente se quiebra en las obras paradigmáticas de Kafka, Rulfo y Beckett. 
Una voluntad que reúne el testimonio negativo de los poderes totalitarios modernos y de sus instrumentos de destrucción: Guernika de Picasso o la Sinfonía Leningrad de Shostakovich. Y que define, al mismo tiempo y en el mismo lugar, el orden ideal de la fantasía humana y del cosmos. La teoría literaria y la teoría estética es la revelación del sentido último de la obra de arte como voluntad de aquel esclarecimiento que más pueda aproximarnos a este sentido a la vez reflexivo y afirmativo de una liberación y una verdad.

\section{Bibliografía}

Beckett, S. (1969). Molloy. Barcelona: Lumen.

Cechinel, A. (2016). O lugar da teoria literaria. Brasil: EDUFSC.

De Andrade, M. (2004). Macunaíma. Barcelona: Octaedro.

Dostoievsky, F. (2014). El idiota. Barcelona: DeBolsillo.

Kafka, F. (2000) El proceso. España: Alianza Editorial.

Mann, T. (1956). Confesiones del estafador Félix Krull. Buenos Aires: Sudamericana.

Picasso, P. (1937). Guernica. Madrid, Museo Nacional Centro de Arte Reina Sofía.

Rulfo, J. (1955). Pedro Páramo. México: Fondo de Cultura Económica.

Shostakóvich, D. (2016). Sinfonía ${ }^{\circ}{ }^{\circ}$ 7, Leningrado. Kúibyshev.

Valmiki. (2000). Ramayana. París: Librería de la Vda de Ch. Bouret. 